Iraqi Journal of Industrial Research (IJOIR) Journal homepage: http://ijoir.gov.iq

\title{
Analyzing of UV Index with the Time Variation for Baghdad
}

\author{
${ }^{1}$ Ahmed A. Alwan*, ${ }^{2}$ Ahmed A. Hameed, ${ }^{1}$ Nuha T. Hamad \\ ${ }^{1}$ Renewable Energy and Environment Research Center/ corporation of industrial research and development, \\ Ministry of Industry and Minerals - Iraq \\ ${ }^{2}$ Space and Communications/ Ministry of Science and Technology - Iraq
}

\section{Article information}

\section{Article history:}

Received: April, 13, 2021

Accepted: April, 24, 2021

Available online: June, 14, 2021

\section{Keywords:}

UV index,

UV radiation,

Solar zenith angle

*Corresponding Author:

Ahmed A. Alwan

ahmedqc22@gmail.com

DOI:

https://doi.org/10.53523/ijoirVol8I1ID32

\begin{abstract}
In order to better realize the effects of UV index (UVI) reaching the earth surface, measurements of effective UVI were carried out during the period of one year over Baghdad; (Lat.33.32- Long.44.45), which receives highly amounts of annual solar radiation. A daily data analysis of UVI is found to reach the highest value during summer reaching the value of 11 , and a minimum in winter with the value of 1 . A relation between UV index and the solar zenith angle was also, it is found that the UVI is highly dependent on the sun elevation where the atmospheric optical path becomes shorter as sun elevation heighten. It can be concluded that Baghdad city exposure to higher amounts of UVI during summer and several hedges must be taken to avoid health harm implications.
\end{abstract}

\section{Introduction}

Solar ultraviolet radiation (UVR) with a high efficient energy could affect all activities of life being on earth and could have a significant impact on human health, ecosystems and materials with such different ways [1]. UVR in the atmosphere comprises $6.12 \%$ of solar spectrum out of the atmosphere, a loss of $2.87 \%$ of incoming solar UV radiation is due to scattering and absorption [2]. UVR can be divided into three essential bands; UVA (315-400) nm, UVB (280-315) nm and UVC (100-280) nm, only UVA and a part of UVB can reach the earth surface, while UVC is totally absorbed by the ozone layer [3]. Increasing the amounts of UVR could have a direct impact on human health, such as melanoma, and skin cancer; also it could also affect photosynthesis of plants and would disrupt aquatic ecosystem.

\section{Theoretical Part}

The Ultraviolet radiation that reaches the Earth's surface showed an increment in recent years at all latitudes except for the equatorial zone. These changes in the amount of UVR caused by ozone depletion, clouds and atmospheric particles are estimated using an equation based on Beer's law for monochromatic and action spectrum weighted irradiances [4]. The UV index is defined as the intensity of UVR reaching the earth's surface that could affect human health. UV radiation levels vary with the time of day and year. Every 1000 meters increase in altitude, UV radiation levels increase by $10 \%$ to $12 \%$, UVI is a unitless quantity and given by the formula [3]: 
$I_{U V}=k_{e r} \cdot \int_{290}^{400} E_{\lambda} \cdot S_{e r}(\lambda) d$

Where $\mathrm{E}_{\lambda}$ is the solar spectral irradiance in $\mathrm{W} /\left(\mathrm{m}^{2} . \mathrm{nm}\right)$ at wavelength $\lambda$, $S_{\text {er }}(\lambda)$ is the erythemal reference action spectrum with a wavelength interval $d \lambda$, and $k_{\text {er }}$ is a constant equal to 40 $\mathrm{m}^{2} / \mathrm{W}$. The erythemal action spectrum coefficient $S_{e r}(\lambda)$ values are given by [5]:

$$
S_{e r}=\left\{\begin{array}{lcc}
1 & \text { for } & \lambda \leq 298 \mathrm{~nm} \\
(10)^{0.094\left(298-\frac{\lambda}{\mathrm{nm}}\right)} & \text { for } 298 \mathrm{~nm}<\lambda \leq 328 \mathrm{~nm} \\
(10)^{0.015\left(139-\frac{\lambda}{\mathrm{nm}}\right)} & \text { for } 328 \mathrm{~nm}<\lambda \leq 400 \mathrm{~nm} \\
0 & \text { for } & \lambda>400 \mathrm{~nm}
\end{array}\right\}
$$

UV Index formulated using the spectrum of erythematic action induced by ultraviolet radiation on human skin of the CIE (International Commission on Illumination), is a simple index given of the risk of direct impact ultraviolet radiation [6].

The index was adopted as a standard indicator of UV levels by the World Meteorological Organization (WMO) and World Health Organization (WHO) in 1994, was based on the erythemal (skin reddening) action spectrum, since this has the most immediate short-term impact on humans. The UV Index was designed to represent erythemal weighted UV radiation in a simple form, as a single number, and it is an irradiance scale computed by multiplying the erythemal irradiance in watts $/ \mathrm{m}^{2}$, by 40 [7].

UVI ranging from 1 to $11+$ according to the exposure intensity effect during the day depends on the solar zenith angle (SZA), the higher the sun in the sky the higher the UVI level; Each category assigned to a color could refer to the range of the UVI intensity as shown in Table (1).

Current guidance on usage of the UV Index for risk prediction extends to values from 1 to $11+$ and establishes five categories of exposure: "low" (1-2), "moderate" (3 -5), "high" (6-7), "very high" (8 -10), and "extreme" (11 or greater). Factors that affect the amount of solar UVR reach the earth's surface (and hence the UV Index) include season, time of day, geographical location, environment (altitude, latitude, and reflective surfaces), atmospheric ozone levels, and cloud scatter of UVR [8].

Table 1: The UVI exposure categories.

\begin{tabular}{|c|c|c|l|}
\hline UVI range & Exposure Category & Colors Signe & Recommendation \\
\hline $1-2$ & Low & Green & No protection \\
\hline $3-5$ & Moderate & Yellow & Protection required \\
\hline $6-7$ & High & Orange & Protection required \\
\hline $8-10$ & Very high & Red & Extra protection \\
\hline $11+$ & Extreme & Violet & Extra protection \\
\hline
\end{tabular}

In this research, a data set of one year of UVI was carried out and analyzed for Baghdad city to illustrate the variation of intensity through a year to collect information on the UVI levels that reach to the top of earth's surface in Baghdad. Such analyses are not available in a literature up to our knowledge.

\section{Experimental Work}

A set of data were taken from Aura/OMI satellite products for one year for Baghdad city (latitude $32.33^{\circ}$, Longitude $44.45^{\circ}$ ), where daily measurements are figured and analyzed. The number of the days with the same value of UVI which occurred through the year distributed according to the intensity levels of UVI for each season is shown in Table (2). 
Table 2: The seasonal distributions of UVI.

\begin{tabular}{|l|c|c|c|c|}
\hline \multicolumn{1}{|c|}{ UV Index } & Winter & Spring & Summer & Autumn \\
\hline Low & 3 & - & - & 6 \\
\hline Medium & 53 & 1 & - & 48 \\
\hline High & 28 & 13 & - & 31 \\
\hline Very High & 5 & 42 & 11 & 35 \\
\hline Extreme & - & 46 & 81 & - \\
\hline
\end{tabular}

Figure (1) illustrates the distribution of UVI values for the days of the same values through the year. It is clear that the extreme values(Add are ) distributed on 6 months a year, the very high distributed on 8 months, high values distributed on 7 months, the medium distributed on 7 months also and the low values distributed on 5 months only.

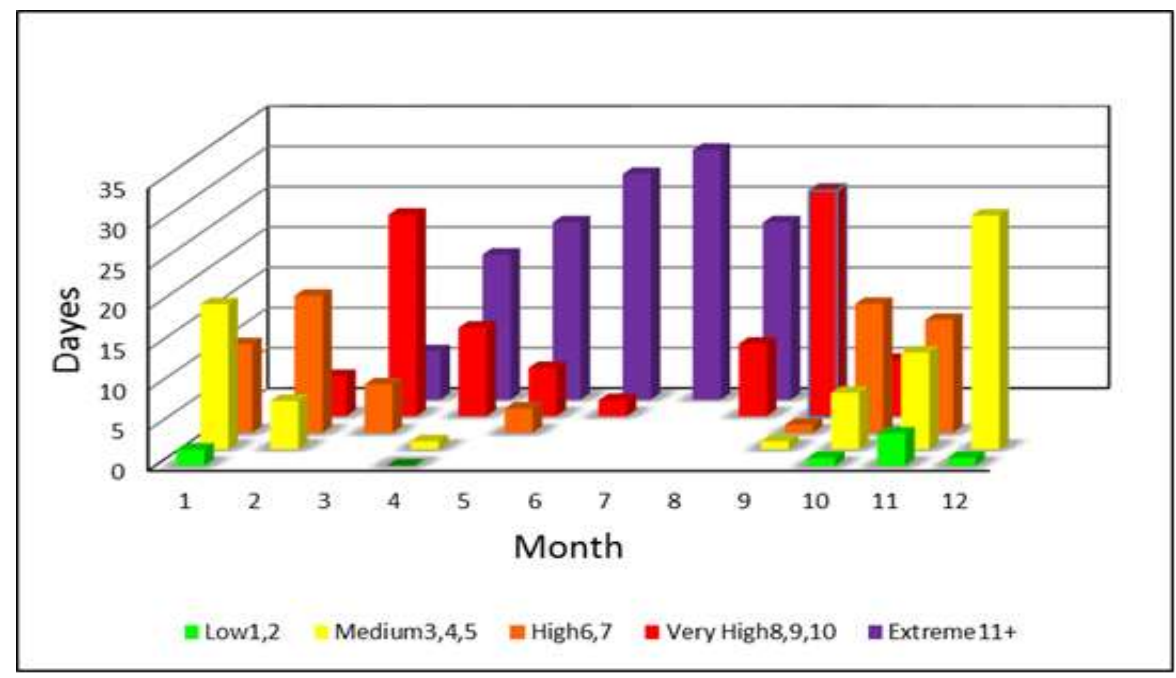

Figure 1: The monthly variation of daily UVI through a year.

Figure (2) shows the plot of UVI values for each day of the year at solar noon time which represents the maximum value. The $4^{\text {th }}$ order polynomial curve is to illustrate the normal behaviour trend of the UVI through a year; it is clear that the maximum lies around the mid-days of the year while the minimum lies in the beginning and last days.

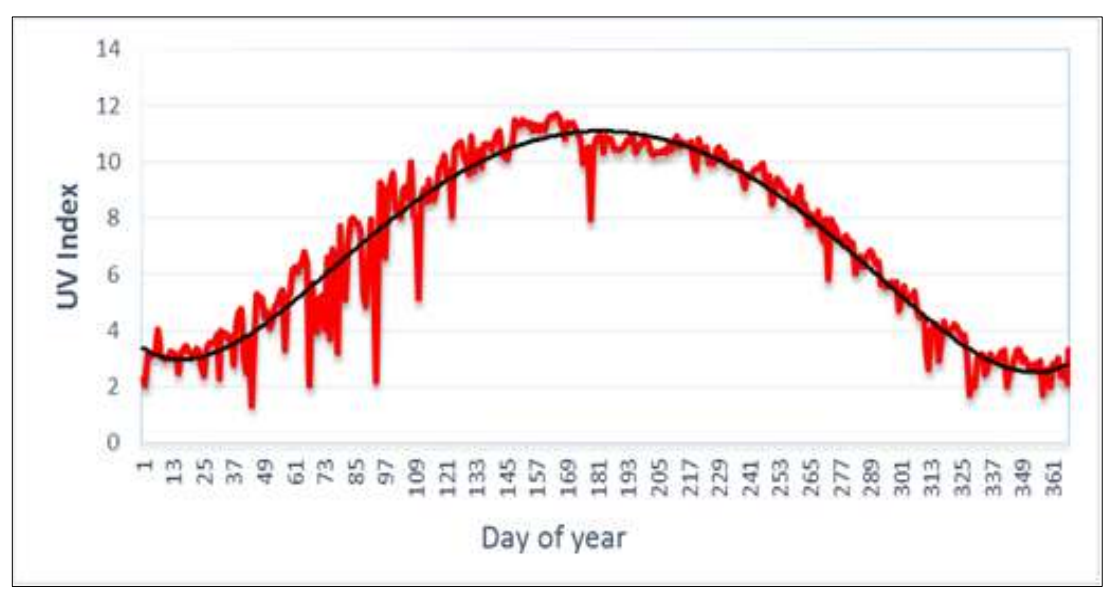

Figure 2: UVI values at solar noon for each day a year. 


\section{Results and Discussion UVI distribution:}

Figure (3) shows the percentage and the number of the days of UVI levels for one year, it is clear that there is(are) 127 days with extreme values which form $34 \%$ of the total amount, while the lowest of UVI is only 8 days which form $2 \%$.

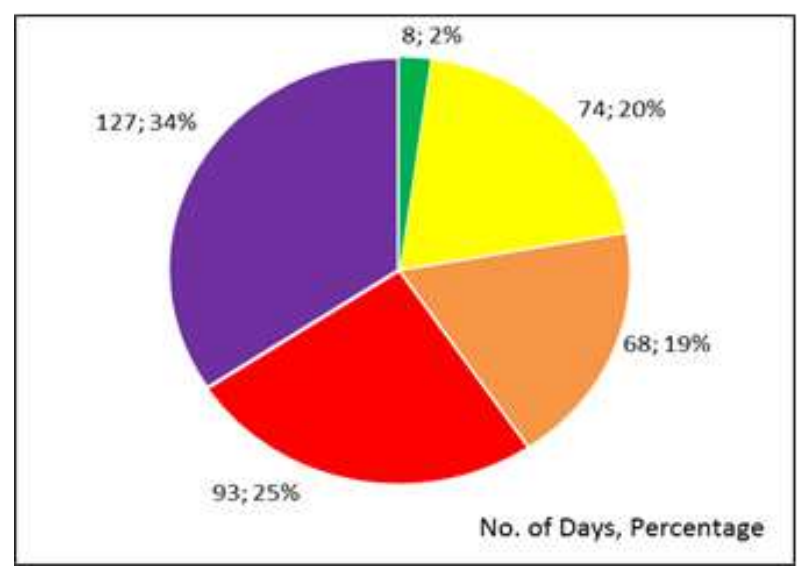

Figure 3: Number of the days and the percentage of UVI levels.

\section{Variation in daily UV index:}

Most of the variability of UVI radiation during the year is attributed to the solar zenith angle (SZA) as shown in Figure (4). In lower angles, photons have a longer path through ozone layer, and this could increasee the absorption. The enhanced probability of interaction with air molecules leads to absorption or backscattering into the atmosphere, this led to attenuate UV radiation at the surface.

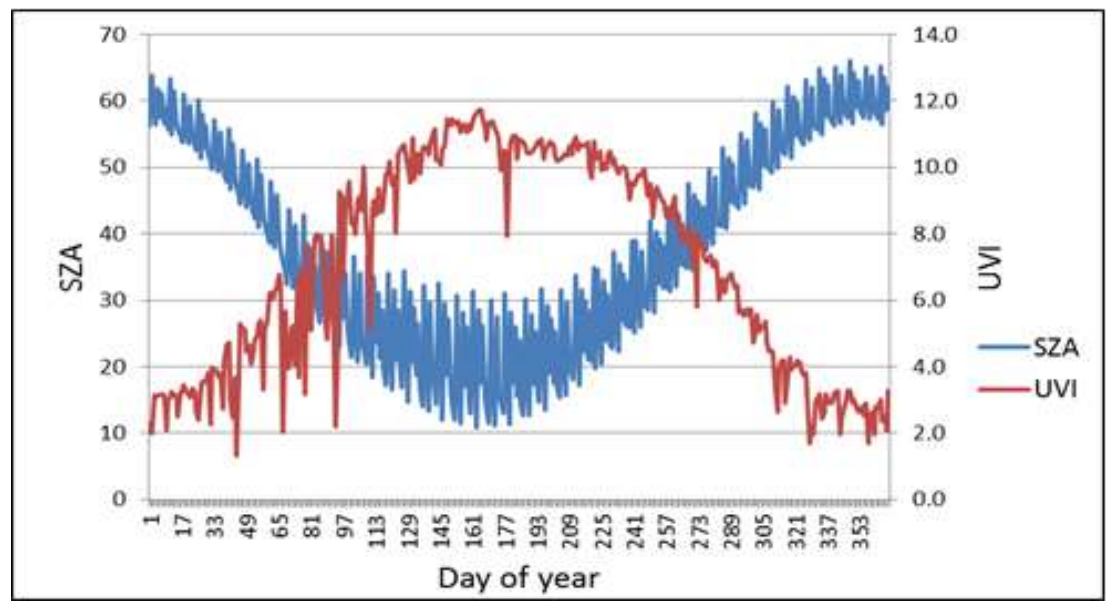

Figure 4: The relation between UVI with the SZA.

\section{Conclusions}

It could be concluded that the UVI reached the highest values in July at mid-day (solar noon) and a minimum in January at the same time. The extreme values of UVI recorded within 127 days, which means an action of warning must be applied to the daily weather forecasting to avoid the skin harmful. A strong relation between SZA and UVI was found; when the UVI increases it could be reached to its maximum value when the sun is being in a vertical position on the top of the earth's surface. Finally several procedures must be taking to avoid UVI exposure especially in the summer months, which could reduce human health risks, and one of the most important priorities that's not be exposure to direct sunlight in the summer sunny days during the period from 10AM to 15PM. 
Acknowledgement: We would like to appreciate the kind of Aura/OMI data bank for providing the necessary data for this research in order to give an accurate result for Baghdad city forecasting based on a satellite observation.

\section{References}

[1] A. V. Lindfors et al., "The TROPOMI surface UV algorithm,” Atmos. Meas. Tech., vol. 11, no. 2, pp. 997-1008, Feb. 2018.

[2] M. Al-Riahi and A. Al-Kayssi, "Some comments on time variation in solar radiation over Baghdad, Iraq," Renew. Energy, vol. 14, no. 1-4, pp. 479-484, May 1998.

[3]E. Rehfuess and World Health Organization., Global solar UV index: a practical guide. World Health Organization, 2002.

[4] J. R. Herman, "Global increase in UV irradiance during the past 30 years (1979-2008) estimated from satellite data," J. Geophys. Res., vol. 115, no. D4, p. D04203, Feb. 2010.

[5] J. F. Sánchez-Pérez, D. Vicente-Agullo, M. Barberá, E. Castro-Rodríguez, and M. Cánovas, "Relationship between ultraviolet index (UVI) and first-, second- and third-degree sunburn using the Probit methodology," Sci. Rep., vol. 9, no. 1, p. 733, Dec. 2019.

[6]CIE, RATIONALIZING NOMENCLATURE FOR UV DOSES AND EFFECTS ON HUMANS Joint publication of CIE and WMO (World Meteorological Organization). WMO, 2014.

[7] V. Fioletov, J. B. Kerr, and A. Fergusson, "The UV index: definition, distribution and factors affecting it.," Can. J. Public Health, vol. 101, no. 4, pp. I5-9, 2010.

[8]P. Gies, E. van Deventer, A. C. Green, C. Sinclair, and R. Tinker, "Review of the Global Solar UV Index 2015 Workshop Report.," Health Phys., vol. 114, no. 1, pp. 84-90, 2018. 